\title{
A comparison between ventricular and lumbar cerebrospinal fluid cytology in adult patients with leptomeningeal metastases
}

\author{
Marc C. Chamberlain, ${ }^{1}$ Patty A. Kormanik, and Michael J. Glantz \\ Kaiser Permanente Medical Group, Department of Neurology, Baldwin Park, CA 91706 (M.C.C.); \\ Department of Nursing, University of California at San Diego, San Diego, CA 92103 (P.A.K.); and \\ University of Massachusetts, School of Public Health and Health Sciences, Amherst, MA 01003 (M.J.G.)
}

Leptomeningeal metastases (LMs) are common metastatic complications, occurring in at least $5 \%$ of patients with disseminated cancer. Cerebrospinal fluid (CSF) cytology remains the standard for diagnosis and assessment of treatment response, but may be inadequate. Our objective was to compare ventricular and lumbar CSF cytology in patients who had cytologically proven LM and were receiving intra-CSF chemotherapy. Sixty patients with LM, positive lumbar CSF cytology documented at diagnosis, limited extent of CNS disease, and no evidence of CSF flow obstruction were treated with a variety of intra-CSF chemotherapies. All patients underwent a single simultaneous ventricular and lumbar CSF sampling (mean volume of CSF per site examined, $10 \mathrm{ml}$ ) to assess response to therapy at either 1 or 2 months after treatment initiation. Ventricular CSF cytology was positive in 44 patients $(73 \%), 35$ of whom were also positive by lumbar CSF cytology. Lumbar CSF cytology was positive in 45 patients $(75 \%)$, of which 35 were also positive by ventricular CSF cytology. Samples were negative at both ventricular and lumbar sites in 6 patients $(10 \%)$. Paired CSF cytologies were discordant in 19 (32\%) patients. The lumbar cytology was negative in 9 , whereas the ventricular cytology was positive (lumbar false-negative rate of $\mathbf{1 7 \%}$ ); the ventricular cytology was negative in 10 , whereas the lumbar cytology was positive (ventricular false-negative rate of $20 \%$ ). In the presence of spinal signs or symptoms of LM, the lumbar CSF cytol-

Received 6 June 2000, accepted 17 August 2000.

${ }^{1}$ Address correspondence and reprint requests to Marc $C$. Chamberlain, Kaiser Permanente Medical Group, Department of Neurology, 1011 Baldwin Park Blvd., Baldwin Park, CA 91706.

${ }^{2}$ Abbreviations used are as follows: CSF, cerebrospinal fluid; LM, leptomeningeal metastasis. ogy was more likely to be positive than was the ventricular (odds ratio $=2.86$; 95\% confidence interval, 0.86 9.56). Conversely, in the presence of cranial signs or symptoms, the ventricular CSF cytology was more likely to be positive than was the lumbar (odds ratio $=2.71$; $95 \%$ confidence interval, 0.76-9.71). In this cohort of patients, whose LM was documented initially by positive lumbar CSF cytology, ventricular and lumbar CSF samples obtained during treatment had similar false-negative rates, depending on the site of clinical or radiologic disease. This suggests that both lumbar and ventricular sites must be sampled when assessing treatment response. If clinical or radiographic disease is present only at 1 site, then CSF from that site is more likely to be positive than is CSF obtained from the more distant site. Neuro-Oncology 3, 42-45, 2001 (Posted to Neuro-Oncology [serial online], Doc. 00-033, October 19, 2000. URL <neurooncology.mc.duke.edu>)

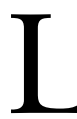

$\mathrm{M}^{2}$ is a common problem for both the neurologist and oncologist (Chamberlain, 1998a; Freilich et al., 1995; Glantz et al., 1999) in treating cancer patients. Several clinical series have estimated LM to occur in $4 \%$ to $15 \%$ of patients with solid tumors, $7 \%$ to $15 \%$ of patients with lymphomas, $5 \%$ to $15 \%$ of patients with leukemia, and $1 \%$ to $2 \%$ of patients with primary brain tumors (Chamberlain, 1998a; Freilich et al., 1995; Glantz et al., 1999).

The single most useful laboratory test in diagnosing LM is an examination of the CSF, usually obtained by lumbar puncture (Bigner, 1998; Chamberlain, 1998a); Freilich et al., 1995; Gajjar et al., 1999; Glantz et al., 1998, 1999; Glass et al., 1979; Russack et al., 1993). In nearly all patients with LM, the CSF is abnormal regardless of the results of CSF cytology. CSF cytology positive for malignant cells is the standard method in most clini- 
cal series by which LM is diagnosed (Bigner, 1998; Gajjar et al., 1999; Glantz et al., 1998; Glass et al., 1979; Russack et al., 1993).

In this retrospective study, 60 adult patients with LM, defined by positive lumbar CSF cytology, and with limited clinical extent of CNS disease, underwent combined lumbar and ventricular CSF sampling to determine response to therapy. CSF samples were compared to determine the utility of single-site sampling and the influence of the disease site (cranial or spinal) on the likelihood of a positive CSF cytology from either site.

\section{Patients and Methods}

\section{Study Population}

Sixty patients ( 32 women and 28 men) aged 19 through 81 years (median 51) with pathologically documented cancer developed LM documented by positive lumbar CSF cytology. All patients were seen by one of the authors (M.C.C., M.J.G.) between August 1986 and December 1999. This patient cohort is a subset of all patients with LM seen by the authors and includes all patients diagnosed during this time period who consented to simultaneous lumbar and ventricular CSF sampling during treatment. All data were collected prospectively and analyzed retrospectively. Tumor histologies included the following: breast cancer $(n=14 ; 23 \%)$; lymphoma $(n=13 ; 22 \%)$; lung cancer $(n=11 ; 18 \%)$; high-grade glioma $(n=6 ; 10 \%)$; medulloblastoma $(n=$ $5 ; 8 \%)$; colon cancer $(n=4 ; 7 \%)$; melanoma $(n=1 ; 2 \%)$; ependymoma $1(n=1 ; 1 \%)$; ovarian cancer $(n=1 ; 3 \%)$; germ cell tumor $(n=1 ; 3 \%)$; and renal cell tumor $(n=$ $1 ; 3 \%)$. Fifty patients $(83 \%)$ had active systemic disease. In 10 patients $(17 \%)$, systemic disease was either stable $(11 \%)$ or in remission $(6 \%)$. Thirty-three patients $(55 \%)$ were treated with a variety of tumor-specific systemic chemotherapies. In addition, all patients were treated with a variety of LM-directed regional chemotherapies by way of an Ommaya reservoir.

Patients presented with symptoms and signs referable to the following neurologic domains: spinal cord $(n=31$; $52 \%)$; cerebral hemispheres $(n=16 ; 27 \%)$; cranial nerves $(n=8 ; 23 \%)$; multiple sites $(n=4 ; 7 \%)$; and no sites $(n=$ $1 ; 2 \%)$. Eighty-five percent of patients were ambulatory; $15 \%$ were nonambulatory, of whom one-half $(8 \%)$ were weight-bearing and walked only with assistance, and onehalf $(7 \%)$ were nonweight-bearing. All nonambulatory patients had either cauda equina $(n=4 ; 10 \%)$ or conus medullaris $(n=2 ; 5 \%)$ syndromes. Karnofsky performance status ranged from 50 to 100 with a mean of 85 .

\section{Imaging}

As previously described, all patients underwent contrast-enhanced cranial and spinal MRI before initiation of LM-directed therapy (Chamberlain, 1998a, 1998b; Chamberlain and Kormanik, 1997; Freilich et al., 1995; Glantz et al., 1999).

In addition, all patients underwent ventricular radioisotopic CSF flow studies before the initiation of
LM-directed therapy (Chamberlain, 1998b; Freilich et al., 1995; Glantz et al., 1999; Chamberlain, 1997; Glantz et al., 1998). In all patients, CSF flow studies demonstrated no evidence of CSF compartmentalization.

\section{CSF Cytology}

All patients were initially diagnosed with LM by a lumbar puncture positive for malignant cells. After diagnosis of LM, patients were neuroradiographically evaluated as indicated above, and an intraventricular catheter and Ommaya reservoir were placed for administering intraCSF chemotherapy. Patients were treated with a variety of intra-CSF chemotherapies, and response was evaluated by serial CSF cytology. Consent for all procedures was obtained according to institutional requirements. As part of treatment evaluation, combined lumbar and ventricular cytologies were obtained within $24 \mathrm{~h}$ (range, 1-24 $\mathrm{h}$; median, $1 \mathrm{~h}$ ). The median volume of CSF obtained from each site for cytological analysis was $10 \mathrm{ml}$ ranging from 8 to $20 \mathrm{ml}$. CSF cytology was cytoprepped the same day in a similar manner (cytospin, Wright-Giemsa stain) and reviewed by the same institutional cytopathologist for the detection of malignant cells (Bigner, 1998; Glantz et al., 1998; Glass et al., 1979).

\section{Results}

Forty-five CSF specimens (75\%) from the lumbar site were positive for malignant cells, and 44 samples $(73 \%)$ from the ventricular site were positive for malignant cells (Table 1). This difference was not statistically significant. Six CSF samples $(10 \%)$ were negative from both the ventricular and lumbar sites. Ten CSF samples from the lumbar site were positive for malignant cytology and negative by ventricular sampling, resulting in a ventricular false-negative rate of $19 \%$. Nine CSF samples were positive by ventricular cytology and negative by concurrent lumbar sampling, resulting in a lumbar false-negative rate of $17 \%$.

The site of the LMs had an appreciable influence on the results of CSF cytology (Table 2). In patients with intracranial disease (defined as either cerebral hemisphere or cranial nerve involvement determined clinically or neuroradiographically), 19 of 24 (79\%) ventricular CSF samples were positive compared with 14 of $24(58 \%)$ lumbar samples (odds ratio $=2.71 ; P=0.12 ; 95 \%$ confidence interval, 0.76-9.71). In patients with involvement of the spinal cord, 26 of 31 (84\%) lumbar samples were positive compared with 20 of $31(65 \%)$ ventricular samples (odds

Table 1. Comparison of simultaneous ventricular and lumbar CSF cytology in 60 patients with LM

\begin{tabular}{lccc} 
& \multicolumn{2}{c}{ Ventricular } & \\
\cline { 2 - 3 } & Positive & Negative & Total \\
\hline Lumbar & 35 & 10 & 45 \\
Positive & 9 & 6 & 15 \\
Negative & 44 & 16 & 60 \\
\hline
\end{tabular}


Table 2. Comparison of location of LMs and site of CSF sampling in 60 patients with LM

Location of LMs

\begin{tabular}{lcccc} 
Sampling site & Cranial (24 paired samples) & Spinal (31 paired samples) & Both (4 paired samples) & Total (59 paired samples) \\
\hline Lumbar & 14 & 26 & 4 & 44 \\
Positive & 10 & 5 & 0 & 15 \\
Negative & 19 & 20 & 4 & 43 \\
Ventricular & 5 & 11 & 0 & 16 \\
Positive & & & \\
Negative & &
\end{tabular}

${ }^{a}$ One patient had a normal neurological examination.

ratio $=2.86 ; P=0.082 ; 95 \%$ confidence interval, 0.86 9.56). In the 4 patients with both cranial and spinal involvement, both ventricular and lumbar CSF cytologies were positive.

\section{Discussion}

This retrospective study highlights several important features regarding cytologic evaluation in patients with LM. Although CSF cytology is the standard marker of leptomeningeal disease, important limitations exist.

As Glass et al. (1979) showed in a postmortem analysis, approximately $40 \%$ of patients with autopsy-proven LMs have negative antemortem CSF cytology. Furthermore, in patients with focal (limited extent) leptomeningeal disease, as many as $50 \%$ to $60 \%$ of patients have negative CSF cytology. In the present study, when only cranial signs or symptoms of LM were present, the ventricular CSF cytology was nearly 3 times more likely to be positive than the lumbar CSF cytology (odds ratio $=2.71 ; P=0.12$ ). Conversely, when only spinal signs or symptoms were present, the lumbar CSF cytology was nearly 3 times more likely to be positive than the ventricular CSF cytology (odds ratio $=2.86 ; P=0.082$ ). Even though 60 is a relatively large number of patients for a clinical series in this disease, that number is too small to permit the $P$ values associated with these odds ratios to reach statistical significance at the 0.05 level. The small numbers also account for the relatively wide 95\% confidence intervals. Nevertheless, these data do suggest that the localization of leptomeningeal disease should direct the site of CSF sampling in order to maximize the diagnostic yield of CSF cytology.

The appropriate volume for CSF cytologic analysis is not widely appreciated, thereby compromising its utility (Glantz et al., 1998). Glantz indicated that a minimum of $10 \mathrm{ml}$ of CSF is optimal for assessing CSF cytology. In a study by Gajjar comparing CSF lumbar and ventricular cytology in children with primary brain tumors, ventriculoperitoneal shunts, and diagnostic staging, the median CSF volume analyzed was $1.0 \mathrm{ml}$ drawn from a venticuloperitoneal shunt as compared with CSF obtained from a lumbar puncture (Gajjar et al., 1999). In the present study, a median of $10 \mathrm{ml}$ of CSF was drawn from both ventricular and lumbar sites. We believe the nearly 10 -fold increase in CSF volume used for cytology evaluation in this study accounts for the equivalency of false-negative rates from both sites as contrasted with the study by Gajjar, which favored lumbar cytology.

Ultimately, the meaning of a single CSF cytology sample is unclear (Freilich et al., 1995; Gajjar et al., 1999; Glantz et al., 1998, 1999; Glass et al., 1979; Russack et al., 1993). In patients with progressive neurologic disease secondary to LM, a single negative CSF cytology sample clearly does not indicate disease control. The meaning of a positive CSF cytology sample in a patient who has LM and is clinically stable is also unclear, and there is no consensus on how to manage such patients. Some neuro-oncologists would continue present LMdirected therapy, and others would change to an alternative therapy based on the CSF cytology. Quantitative CSF cytology may help in this regard, because a documented decrease in the number of malignant cells suggests a partial response to LM-directed therapies (Russack et al., 1993). Furthermore, a single negative CSF cytology, even in a clinically stable patient, does not necessarily indicate treatment response. Rather, this finding should be confirmed by subsequent CSF analysis at all sites previously reported as positive (Chamberlain, 1998a; Glantz et al., 1999). The optimal interval between response-defining CSF cytologies has varied from 1 to 4 weeks, and no consensus yet exists (Chamberlain, 1998a; Glantz et al., 1998).

In conclusion, patients with cytologically defined LM may be evaluated for response to treatment by either lumbar or ventricular CSF sampling with similar false-negative cytology rates. The rate of false-negative CSF cytology is in part dependent on the site and extent of leptomeningeal disease, even in patients with unobstructed CSF flow. We believe that adequate assessment of CSF cytologic response requires CSF samples from both lumbar and ventricular sites, assuming a ventricular catheter and reservoir are present. Particular emphasis should be placed on examining CSF from sites of clinical or neuroradiographic disease, as these are more likely to be cytologically positive. Though not directly studied, the findings in this study also suggest that the initial clinical or radiographic diagnosis of LM is more likely to be confirmed by CSF sampling directed at the site of clinical or neuroradiographic disease (Glantz et al., 1998; Rogers et al., 1992). These findings have implications for both the clinical management and the design and interpretation of clinical trials involving patients with LM. 


\section{References}

Bigner, S.H. (1998) Cerebrospinal fluid: Cytologic interpretation. In Bigner, D.D., McLendon, R.E., and Brunner, J.M. (Eds.), Russell and Rubinstein's Pathology of Tumors of the Nervous System. Sixth edition. London: Amold. pp. 681-702.

Chamberlain, M.C. (1998a) Leptomeningeal metastases: A review of evaluation and treatment. J. Neurooncol. 37, 271-284.

Chamberlain, M.C. (1998b) Radioisotope CSF flow studies in leptomeningeal metastases. J. Neurooncol. 38, 135-140.

Chamberlain, M.C., and Kormanik, P.A. (1997) Prognostic significance of coexistent bulky metastatic central nervous system disease in patients with leptomeningeal metastases. Arch. Neurol. 54, 1364-1368.

Freilich, R.J., Krol, G., and DeAngelis, L.M. (1995) Neuroimaging and cerebrospinal fluid cytology in the diagnosis of leptomeningeal metastasis. Ann. Neurol. 38, 51-57.

Gajjar, A., Fouladi, M., Walter, A.W., Thompson, S.J., Reardon, D.A., Merchant, T.E., Jenkins, J.J., Liu, A., Boyett, J.M., Kun, L.E., and Heideman, R.L. (1999) Comparison of lumbar and shunt cerebrospinal fluid specimens for cytologic detection of leptomeningeal disease in pediatric patients with brain tumors. J. Clin. Oncol. 17, 1825-1828.
Glantz, M.J., Cole, B.F., Glantz L.K., Cobb, J., Mills, P., Lekos, A., Walters, B.C., and Recht, L.D. (1998) Cerebrospinal fluid cytology in patients with cancer: Minimizing false-negative results. Cancer 82, 733-739.

Glantz, M.J., LaFollette, S., Jaeckle, K.A., Shapiro, W., Swinnen, L., Rozental, J.R., Phuphanich, S., Rogers, L.R., Gutheil, J.C., Batchelor, T., Lyter, D., Chamberlain, M., Maris, B.L., Schiffer, C., Bashir, R., Thomas, D., Cowens, W., and Howell, S.B. (1999) Randomized trial of a slow-release versus a standard formulation of cytarabine for the treatment of lymphomatous meningitis. J. Clin. Oncol. 17, 3110-3116.

Glass, J.P., Melamed, M., Chernik, N.L., and Posner, J.B. (1979) Malignant cells in cerebrospinal (CSF): The meaning of a positive CSF cytology. Neurology 29, 1369-1375.

Russack, V., Kim, S., and Chamberlain, M.C. (1993) Quantitative cerebrospinal fluid cytology in patients receiving intracavitary chemotherapy. Ann. Neurol. 34, 108-112.

Rogers, L.R., Duchesneau, P.M., Nunez, C., Fishleder, A.J., Weick, J.K., Bauer, L.J., and Boyett, J.M. (1992) Comparison of cisternal and lumbar CSF examination in leptomeningeal metastasis. Neurology 42, 1239-1241. 